\title{
ATRIBUTOS BIOLÓGICOS E DINÂMICA DA MATÉRIA ORGÂNICA EM LATOSSOLOS AMARELOS NA REGIÃO DO CERRADO PIAUIENSE SOB SISTEMA PLANTIO DIRETO $^{(1)}$
}

\author{
Rossanna Barbosa Pragana( ${ }^{(2)}$, Rafaela Simão Abrahão Nóbrega(3), \\ Mateus Rosas Ribeiro(4) \& José Ferreira Lustosa Filho(5)
}

\begin{abstract}
RESUMO
A utilização do solo sob Cerrado tem acarretado modificações nas suas propriedades, bem como no comportamento e qualidade da sua matéria orgânica. O Cerrado piauiense vem sendo alvo de exploração sem a devida preocupação com a manutenção dos recursos naturais, onde os sistemas de produção têm se caracterizado pelo uso intensivo do solo. O objetivo desta pesquisa foi avaliar o efeito da utilização agrícola na alteração dos atributos biológicos e na dinâmica da matéria orgânica em Latossolos Amarelos distróficos típicos, cultivados com soja. O trabalho foi realizado na Serra do Quilombo, localizada no Cerrado piauiense. Foram verificadas as alterações nos atributos do solo em decorrência da utilização agrícola, que foi iniciada com plantio convencional, posteriormente substituído pelo sistema plantio direto. Foram amostrados solos com diferentes históricos de uso: PC7/PD8 - sete anos de plantio convencional e oito de plantio direto; PC5/PD4 - cinco anos de plantio convencional e quatro de plantio direto; PC3/PD3 - três anos de plantio convencional e três de plantio direto; e CN - solo com Cerrado nativo. Os atributos avaliados foram: respiração basal do solo, carbono da biomassa microbiana, quociente metabólico, quociente microbiano, carbono orgânico total e nitrogênio total. Para avaliar a dinâmica da matéria orgânica, foi feito o fracionamento físico. Os dados foram submetidos à análise de variância. Os resultados indicaram que o manejo imposto ao solo com cultivo
\end{abstract}

(1) Parte da Tese de Doutorado do primeiro autor apresentada à Universidade Federal Rural de Pernambuco - UFRPE. Recebido para publicação em 6 de setembro de 2011 e aprovado em 14 de fevereiro de 2012.

(2) Professora da Unidade Acadêmica de Serra Talhada, Universidade Federal Rural de Pernambuco - UFRPE. Fazenda Saco s/n, Caixa Postal 063, CEP 56900-000 Serra Talhada (PE). E-mail: rossannapragana@yahoo.com.br

(3) Professora do Campus Professora Cinobelina Elvas (CPCE) da UFPI, BR 135 Planalto Cibrazem, CEP 64900-000 Bom Jesus (PI). E-mail: rafaela.nobrega@gmail.com

(4) Professor Associado do Departamento de Agronomia, UFRPE. R. Dom Manoel de Macedo s/n, Dois Irmãos, CEP 52171-900 Recife (PE). Bolsista do CNPq. E-mail: mrosas@depa.ufrpe.br

(5) Graduando em Agronomia, UFPI. Bolsista de Iniciação Científica. E-mail: filhoze04@hotmail.com 
convencional e posterior implantação do sistema plantio direto contribuiu para alterar as propriedades biológicas do solo, em comparação com o Cerrado nativo. A substituição do Cerrado nativo por culturas anuais sob o sistema plantio direto reduz os teores de carbono da biomassa microbiana e de nitrogênio total e eleva a respiração basal nos tratamentos com três e quatro anos de implantação do plantio direto, indicando que esse tempo é insuficiente para melhoria desses atributos. $\mathrm{O}$ aumento do tempo de uso do solo com plantio direto diminui o teor de $\mathrm{C}$ particulado e mantém o de $\mathrm{C}$ associado aos minerais, em comparação ao solo do Cerrado nativo.

Termos de indexação: atividade microbiana, frações orgânicas, sistemas de manejo, uso do solo.

\title{
SUMMARY: EFFECT OF CROPPING SYSTEMS ON BIOLOGICAL PROPERTIES AND ORGANIC MATTER DYNAMICS OF YELLOW OXISOLS IN THE CERRADO REGION OF PIAUÍ, BRAZIL
}

\begin{abstract}
The intensive use of soils in the Cerrado biome has caused modifications in soil properties, as well as in the performance and quality of organic matter. The Cerrado of Piaui has been exploited without due concern for the preservation of natural resources, where production systems are characterized by intensive land use. The objective of this research was to evaluate the effect of the agricultural use in altering the biological properties and dynamics of organic matter of Yellow Oxisols cultivated with soybean. This research was carried out in the "Serra do Quilombo", located in the Cerrado region of Piaui state. To assess changes in soil properties, induced by agricultural use, soil samples were collected from sites with different use history involving conventional and no tillage systems; PC7/PD8 - seven years of conventional agriculture followed by eight years of no-tillage; PC5/PD4-five years of conventional system followed by four years of no-tillage; PC3/PD3-three years of conventional system and three years of no-tillage and $C N$-native cerrado vegetation. The evaluated soil properties were basal respiration, microbial biomass $C$, metabolic quotient, microbial quotient, total organic C and total $N$. Physical fractionation was used to evaluate organic matter dynamics. The results were subjected to variance analysis and showed that the soil management under conventional cultivation and subsequent implantation of notillage system affected soil biological properties in relation to the soil under native cerrado vegetation. The replacement of native cerrado by annual no-tillage crops reduces the levels of microbial biomass $C$ and total $N$ and elevates the basal respiration in treatments after three and four years of no tillage, indicating that these periods are insufficient to improve the above properties. The increase in the time of land use with no tillage decreases the level of particulate organic $C$ and maintains the $C$-associated minerals in relation to the soil under native cerrado vegetation.
\end{abstract}

Index terms: microbial activity, organic fractions, management system, soil use.

\section{INTRODUÇÃO}

A atividade e a população dos organismos no solo estão diretamente relacionadas com o volume de material orgânico disponível, que constitui uma das principais fontes de energia para os organismos. O constante revolvimento da superfície do solo e a falta de cobertura vegetal no sistema convencional contribuem para diminuição das populações de organismos do solo (principalmente microrganismos), embora a mobilização do solo possa elevar em curto prazo a biomassa microbiana, por proporcionar a incorporação de resíduos orgânicos, disponibilizando substrato orgânico com a quebra dos agregados (Ferreira et al., 2007). Por outro lado, devido à maior quantidade de resíduos vegetais depositados na superfície e a outros efeitos sobre os atributos do solo, o sistema plantio direto tende a facilitar um incremento na vida biológica do solo (Calegari, 1998). 
Apesar de pesquisas evidenciarem a ocorrência de melhores condições biológicas do solo sob plantio direto, em comparação com o plantio convencional, Rosa et al. (2003) observaram maiores valores para o C da biomassa microbiana (Cmic) no solo sob floresta nativa, em relação ao plantio direto. A maior diversidade de espécies vegetais encontradas na floresta, que se reflete diretamente numa maior amplitude de organismos vivos, favorecidos pelas condições ambientais do solo, é uma explicação para esse fato. No entanto, Santos et al. (2004) encontraram valores semelhantes de $\mathrm{C}$ da biomassa microbiana comparando solos em plantio direto e em condições naturais, na profundidade de $0-5 \mathrm{~cm}$. Estes autores concluíram que sistemas de manejo que elevam os teores de C no solo proporcionam maior atividade microbiana.

Além do $\mathrm{C}$ da biomassa microbiana, o quociente microbiano ( $q \mathrm{Mic}$ ) é um índice bastante utilizado para fornecer indicações sobre a dinâmica da matéria orgânica, expressando a eficiência da biomassa microbiana em utilizar o carbono orgânico do solo. Esse índice pode variar de acordo com o valor de $\mathrm{pH}$ do solo, sistemas de preparo do solo, quantidade e qualidade do aporte de C (Anderson $\&$ Domsch, 1993). Maior $q$ Mic representa maior ciclagem de nutrientes, por haver maior quantidade de microrganismos em termos de $\mathrm{C}$, quando comparado ao carbono orgânico total.

A disponibilidade de $\mathrm{C}$ no solo tem sido descrita como a fonte que contribui para o aumento da respiração basal do solo (RBS), que é definida como a soma total de todas as funções metabólicas nas quais o $\mathrm{CO}_{2}$ é produzido. É necessário, portanto, entender o efeito de diferentes sistemas de manejo sobre a quantidade da biomassa microbiana do solo e sua ação na liberação e no fornecimento adequado de nutrientes para as culturas no Cerrado (Figueiredo et al., 2007).

$\mathrm{O}$ quociente metabólico ou respiratório $\left(q \mathrm{CO}_{2}\right)$ indica a eficiência da biomassa microbiana em utilizar o $\mathrm{C}$ disponível para biossíntese, sendo sensível indicador para estimar a atividade biológica e a qualidade do substrato (Saviozzi et al., 2002). Segundo Anderson \& Domch (1993), valores de $q \mathrm{CO}_{2}$ elevados são um indicativo de comunidades microbianas em estádios iniciais de desenvolvimento, com maior proporção de microrganismos ativos em relação aos inativos, ou seja, um indicativo de populações microbianas sob algum tipo de estresse metabólico.

As diferentes formas de fracionamento utilizadas nos estudos da matéria orgânica do solo (MOS) tendem a reduzir a heterogeneidade das frações orgânicas, procurando separar frações homogêneas quanto à natureza, dinâmica e função (Christensen,
2000). O fracionamento físico tem se mostrado promissor na distinção dos compartimentos de carbono do solo sujeitos à influência do manejo (Collins et al., 1997), sendo ele subdividido em fracionamento densimétrico e granulométrico. De acordo com Nicoloso (2005), o carbono orgânico particulado (COP) da matéria orgânica do solo constitui importante ferramenta indicadora de qualidade do solo em curtos períodos de avaliação. Assim, o carbono orgânico particulado pode ser utilizado como um indicador de qualidade do solo para avaliação de sistemas de manejo recentes, nos quais as alterações na matéria orgânica ainda não tenham sido de grande magnitude (Conceição et al., 2005), por ser considerado a parte mais lábil do solo, que responde prontamente aos sistemas de manejo. Por ser uma fração lábil e com maior taxa de reciclagem dos constituintes orgânicos, as alterações nos estoques de COP promovidas pelo manejo do solo são percebidas geralmente em curto prazo, em comparação às alterações mais lentas que ocorrem no CO do solo como um todo (Heide et al., 2009). Já o carbono orgânico associado aos minerais é a parte mais estável, não apresentando sensibilidade imediata às alterações nas práticas de manejo, sendo considerado o estoque de carbono a médio e longo prazo (Salton et al., 2005). A alteração do COM é menor pelo fato de este possuir ciclagem mais lenta que a fração COP, em virtude de seu avançado estádio de humificação e estabilidade, conferido pela interação com a fração mineral do solo, localização no interior de microagregados e maior recalcitrância química devido à sua composição (Heide et al., 2009).

O objetivo deste trabalho foi avaliar as alterações nos atributos biológicos e na dinâmica da matéria orgânica em Latossolos Amarelos distróficos típicos cultivados com soja em função de diferentes tempos de implantação do plantio direto, após cultivo anterior em sistema de plantio convencional, confrontando os resultados com as características do solo de uma área adjacente de preservação com Cerrado nativo.

\section{MATERIAL E MÉTODOS}

A área de estudo está localizada na Serra do Quilombo, no município de Bom Jesus-PI, que apresenta clima quente e semiúmido do tipo Aw (Köppen), com temperatura variando de 18 a $36{ }^{\circ} \mathrm{C}$. Na área predominam Latossolos Amarelos distróficos típicos, muito profundos e bem drenados, em relevo plano, cultivados com soja comercial. Foram selecionados talhões sob diferentes tempos de uso com plantio direto (PD) na Fazenda São Marcos. No quadro 1 encontra-se o histórico de uso dos solos das áreas estudadas e a localização geográfica. 
Quadro 1. Histórico de uso e localização de Latossolos Amarelos da região da Serra do Quilombo, no sudoeste do Estado do Piauí

\begin{tabular}{|c|c|}
\hline Símbolo & Histórico \\
\hline $\mathrm{CN}$ & $\begin{array}{l}\text { Vegetação nativa de cerrado, sem histórico de interferência humana em termos de uso agrícola. Coordenadas } \\
09^{\circ} 10^{\prime} 40,85^{\prime \prime} \mathrm{S} \text { e } 45^{\circ} 07^{\prime} 07^{\prime \prime} \mathrm{W} \mathrm{Gr}\end{array}$ \\
\hline PC3/PD3 & $\begin{array}{l}\text { Manejo com plantio convencional (monocultura de soja por quatro anos, } 2003-2005 \text { ). O sistema PD for } \\
\text { implantado a partir de } 2006 \text {, com o cultivo de soja. Coordenadas } 09^{\circ} 11^{\prime} 48,7 \text { " } \mathrm{S} \text { e } 45^{\circ} 08^{\prime} 14,2^{\prime} \text { W } \mathrm{Gr}\end{array}$ \\
\hline PC5/PD4 & $\begin{array}{l}\text { Monocultura de soja desde } 2000 \text {. O sistema PD foi instalado em } 2005 \text {, em rotação anual de soja e milho. } \\
\text { Coordenadas } 09^{\circ} 10^{\prime} 33,9^{\prime} \text { S e } 45^{\circ} 08^{\prime} 20,4 \text { " W Gr }\end{array}$ \\
\hline PC7/PD8 & $\begin{array}{l}\text { Área convertida em sistema agrícola no ano de } 1994 \text {, sendo desmatada e cultivada em sistema plantio } \\
\text { convencional com revolvimento intensivo de solo. O sistema PD foi implantado em } 2002 \text {, com soja, utilizando } \\
\text { o milheto na formação da palhada. Em } 2009 \text { foi introduzida na área forrageira do gênero Brachiaria sp. após } \\
\text { a colheita do milho. Coordenadas } 09^{\circ} 10^{\prime} 48,8^{\prime} \mathrm{S} \text { e } 45^{\circ} 07^{\prime} 46,1^{\prime} \mathrm{W} \text { Gr }\end{array}$ \\
\hline
\end{tabular}

As amostras de solo para avaliação das alterações das características biológicas, coletadas nas profundidades de $0-5$ e 5-10 $\mathrm{cm}$ em cada área estudada, foram acondicionadas em ambiente refrigerado a $4{ }^{\circ} \mathrm{C}$ até a realização das análises, após serem submetidas a peneira de $2,0 \mathrm{~mm}$ de malha.

A determinação da respiração basal do solo (RBS) foi realizada segundo o método descrito por Isermeyer (1952), citado por Alef \& Nannipieri (1995). Para cada amostra de solo, foram retiradas três subamostras de $50 \mathrm{~g}$, que foram umedecidas até teor de água ajustado a $70 \%$ da capacidade de campo. Estas foram acondicionadas em béqueres de $50 \mathrm{~mL}$, os quais foram colocados no fundo de recipientes plásticos de $2 \mathrm{~L}$, juntamente com outro béquer contendo $20 \mathrm{~mL}$ de $\mathrm{NaOH}\left(0,05 \mathrm{~mol} \mathrm{~L}^{-1}\right)$, para capturar o $\mathrm{CO}_{2}$ liberado do solo. Os recipientes plásticos foram vedados hermeticamente e incubados, por $72 \mathrm{~h}$, em temperatura controlada $\left(27 \pm 2{ }^{\circ} \mathrm{C}\right)$. Foram preparados três recipientes controle, com o $\mathrm{NaOH}\left(0,05 \mathrm{~mol} \mathrm{~L}^{-1}\right)$, sem amostra de solo. Após o período de incubação, as soluções de $\mathrm{NaOH}$ receberam $5 \mathrm{~mL}$ de $\mathrm{BaCl}\left(0,5 \mathrm{~mol} \mathrm{~L}^{-1}\right)$ para a precipitação do carbonato e foram imediatamente tituladas com $\mathrm{HCl}\left(0,05 \mathrm{~mol} \mathrm{~L}^{-1}\right)$, após a adição de três gotas de fenolftaleína (1\%) como indicador.

O carbono da biomassa microbiana (Cmic) foi determinado pelo método descrito por Ferreira et al. (1999), utilizando-se o forno de micro-ondas para preparação das amostras irradiadas (potência real do aparelho 767,07 W), por três min, para eliminar os microrganismos, provocar o rompimento da célula e a liberação de compostos intracelulares. $\mathrm{O}$ Cmic foi extraído por uma solução de $\mathrm{K}_{2} \mathrm{SO}_{4}$ $0,5 \mathrm{~mol} \mathrm{~L}^{-1}$. Uma alíquota de $10 \mathrm{~mL}$ do extrato filtrado foi utilizada para essa determinação. A esta alíquota foram acrescentados $2 \mathrm{~mL}$ de $\mathrm{K}_{2} \mathrm{Cr}_{2} \mathrm{O}_{7}$ 0,066 mol L-1 e $10 \mathrm{~mL}$ de $\mathrm{H}_{2} \mathrm{SO}_{4}$ em erlenmeyers de $250 \mathrm{~mL}$. Essa solução foi diluída com $50 \mathrm{~mL}$ de água destilada e resfriada. $\mathrm{O}$ dicromato residual foi quantificado por titulação com uma solução de sulfato ferroso amoniacal $\left[\mathrm{Fe}\left(\mathrm{NH}_{4}\right)_{2}\left(\mathrm{SO}_{4}\right)_{2} 6 \mathrm{H}_{2} \mathrm{O}\right]$ em $\mathrm{H}_{2} \mathrm{SO}_{4}$ concentrado, na presença de uma solução indicadora composta por fenantrolina e sulfato ferroso (Ferroin). A quantidade de Cmic foi determinada pela diferença entre o $\mathrm{C}$ extraído das amostras de solo irradiadas e o das não irradiadas, usando-se fator de correção $\left(\mathrm{k}_{\mathrm{C}}\right)$ de 0,33 .

$\mathrm{O}$ quociente metabólico $\left(q \mathrm{CO}_{2}\right)$ foi obtido pela razão entre o RBS e o Cmic, e o quociente microbiano ( $q \mathrm{Mic}$ ), obtido pela relação entre o Cmic e o $\mathrm{C}$ orgânico total (COT), ambos seguindo o método de Anderson \& Domsch (1993). O N total do solo foi determinado empregando-se o método Kjeldahl. Para determinação do carbono orgânico oxidável, utilizou-se, como agente oxidante, o dicromato de potássio em meio sulfúrico, sendo a reação acelerada por aquecimento. Como titulante, foi utilizado o sulfato ferroso amoniacal.

Para avaliação da dinâmica da matéria orgânica do solo, foi feito o fracionamento físico por gravimetria nas amostras de solo coletadas nos horizontes A e $\mathrm{AB}$, em cada tratamento. Neste trabalho adotou-se o método descrito por Cambardella \& Elliott (1992), utilizando como dispersante uma solução normal de $\mathrm{NaOH}$ na proporção 2:1 (solo:solução), seguido de agitação rápida por $15 \mathrm{~min}$. O C determinado na fração retida na peneira de $53 \mu \mathrm{m}$ foi denominado de carbono orgânico particulado (COP). Os valores de carbono associado aos minerais foram obtidos subtraindo do COT o COP.

O delineamento estatístico utilizado foi inteiramente casualizado (DIC), em parcelas subdivididas, com cinco repetições, em que as parcelas correspondem às áreas com diferentes tempos de implantação do PD e sob Cerrado 
nativo (PC3/PD3 - plantio direto há três anos, PC5/PD4 - plantio direto há quatro anos, PC7/ PD8 - plantio direto há oito anos e CN - Cerrado nativo), e as subparcelas, às profundidades de amostragem, representadas pelos horizontes $\mathrm{A}$ e $\mathrm{AB}$ e profundidades de $0-5$ e $5-10 \mathrm{~cm}$. Os resultados foram submetidos à análise de variância, sendo os efeitos da profundidade, do uso do solo e da interação uso do solo x profundidade comparados pelo teste de Tukey a $5 \%$, empregando-se o programa computacional SAS/STAT (SAS, 2002).

\section{RESULTADOS E DISCUSSÃO}

$\mathrm{Na}$ camada de $0-5 \mathrm{~cm}$, os maiores valores encontrados para respiração basal do solo (RBS) foram nos tratamentos PC5/PD4 e PC3/PD3 (Quadro 2), não diferindo entre si, indicando maior metabolismo dos microrganismos nesses solos. Na camada de 5-10 cm não houve diferença significativa entre os tratamentos. No entanto, os valores da respiração decresceram, diferindo estatisticamente dos da camada de $0-5 \mathrm{~cm}$. Nas camadas superficiais do solo, a atividade microbiana tende a ser maior devido à maior quantidade de matéria orgânica decomposta pela biota do solo. De acordo com Matias et al. (2009), distúrbios no solo podem provocar desvios da energia do crescimento e reprodução dos microrganismos para a manutenção celular, de forma que uma parte do carbono microbiano é perdida na forma de $\mathrm{CO}_{2}$.
Por outro lado, a baixa respiração do solo no $\mathrm{CN}$ e PC7/PD8 pode indicar estabilidade. De acordo com Gama-Rodrigues \& Gama-Rodrigues (2008), quando a biomassa microbiana está em equilíbrio, são observadas menores perdas de $\mathrm{CO}_{2}$ pela respiração, e, com isso, maior é a incorporação de $\mathrm{C}$ à biomassa microbiana.

$\mathrm{O} \mathrm{CN}$ foi o que apresentou menor $q \mathrm{CO}_{2}$, diferindo apenas do tratamento PC5/PD4 (Quadro 2). Este último tratamento apresentou maior $q \mathrm{CO}_{2}$, demonstrando a perda de $\mathrm{C}$ do solo, comprovada pela menor concentração de $\mathrm{C}$ retido na biomassa microbiana (Cmic) e no COT (Quadro 2), ainda que as diferenças entre os tratamentos com solo cultivado não tenham sido significativas quanto a esses atributos. De acordo com Tótola \& Chaer (2002), baixo $q \mathrm{CO}_{2}$ indica economia na utilização de energia e, supostamente, reflete um ambiente mais estável ou próximo do seu estado de equilíbrio. Ao contrário, valores elevados são indicativos de ecossistemas submetidos a alguma condição de estresse ou de distúrbio. Segundo Tu et al. (2006), alta $q \mathrm{CO}_{2}$ pode significar rápida transformação de resíduos orgânicos em nutrientes para as plantas ou pode ser indicativo de estresse sobre a biomassa microbiana pela perturbação do solo, principalmente pelo trânsito de máquinas e implementos agrícolas durante as atividades de colheita e preparo do solo.

$\mathrm{Na}$ profundidade de $0-5 \mathrm{~cm}$, o $\mathrm{CN}$ apresentou teor elevado de Cmic (316,04 $\mathrm{mg} \mathrm{kg}^{-1} \mathrm{de}$ solo), significativamente maior do que os valores obtidos nos demais tratamentos. Interações significativas

Quadro 2. Respiração basal do solo (RBS), carbono da biomassa microbiana (Cmic), quociente metabólico $\left(q \mathrm{CO}_{2}\right)$, quociente microbiano (qMic), nitrogênio total (NT), carbono orgânico total (COT), carbono orgânico particulado (COP) e carbono orgânico associado ao mineral (COM) de Latossolos Amarelos cultivados sob plantio direto e de uma área de Cerrado nativo

\begin{tabular}{|c|c|c|c|c|c|c|c|c|}
\hline Tratamento & RBS & Cmic & $\mathrm{qCO}_{2}$ & qMic & NT & COT & COP & COM \\
\hline & $\mathrm{mg} \mathrm{kg}^{-1} \mathrm{~h}^{-1}$ & $\mathrm{mg} \mathrm{kg}^{-1}$ & $\underset{\text { Cmic dia }}{\mathrm{mg} \mathrm{CO}_{2} \mathrm{mg}^{-1}}$ & $\%$ & & g k & & \\
\hline \multicolumn{6}{|c|}{ Profundidade $0-5 \mathrm{~cm}$} & \multicolumn{3}{|c|}{ Horizonte A } \\
\hline $\mathrm{CN}$ & $1,95 \mathrm{~B} \mathrm{a}$ & $316,04 \mathrm{~A} \mathrm{a}$ & $0,17 \mathrm{~B} \mathrm{a}$ & $1,67 \mathrm{~A} \mathrm{a}$ & $1,99 \mathrm{~A} \mathrm{a}$ & $18,91 \mathrm{~A} \mathrm{a}$ & $3,16 \mathrm{~A} \mathrm{a}$ & $15,81 \mathrm{AB}$ a \\
\hline $\mathrm{PC} 7 / \mathrm{PD} 8$ & $2,28 \mathrm{~B} \mathrm{a}$ & $159,65 \mathrm{~B} \mathrm{a}$ & $0,37 \mathrm{AB}$ a & $0,89 \mathrm{~A} \mathrm{a}$ & $0,94 \mathrm{~B} \mathrm{a}$ & $18,00 \mathrm{AB}$ a & $2,18 \mathrm{~B} \mathrm{a}$ & $15,75 \mathrm{~B} \mathrm{a}$ \\
\hline PC5/PD4 & $3,75 \mathrm{~A} \mathrm{a}$ & $126,69 \mathrm{~B} \mathrm{a}$ & $0,84 \mathrm{~A} \mathrm{a}$ & $1,14 \mathrm{~A} \mathrm{a}$ & $0,97 \mathrm{~B} \mathrm{a}$ & $13,36 \mathrm{~B} \mathrm{a}$ & $2,20 \mathrm{~B} \mathrm{a}$ & $11,16 \mathrm{~B} \mathrm{a}$ \\
\hline PC3/PD3 & $3,69 \mathrm{~A} \mathrm{a}$ & $149,42 \mathrm{~B} \mathrm{a}$ & $0,61 \mathrm{AB}$ a & $0,79 \mathrm{~A} \mathrm{a}$ & $0,98 \mathrm{~B} \mathrm{a}$ & $21,45 \mathrm{~A} \mathrm{a}$ & $2,46 \mathrm{AB}$ a & $18,99 \mathrm{~A} \mathrm{a}$ \\
\hline \multicolumn{6}{|c|}{ Profundidade $5-10 \mathrm{~cm}$} & \multicolumn{3}{|c|}{ Horizonte AB } \\
\hline $\mathrm{CN}$ & $1,04 \mathrm{~A} \mathrm{~b}$ & $193,77 \mathrm{~A} \mathrm{a}$ & $0,13 \mathrm{~A} \mathrm{a}$ & $1,02 \mathrm{~A} \mathrm{a}$ & $1,12 \mathrm{~A} \mathrm{a}$ & $9,86 \mathrm{~A} \mathrm{~b}$ & $0,61 \mathrm{~A} \mathrm{~b}$ & $9,24 \mathrm{~A} \mathrm{~b}$ \\
\hline $\mathrm{PC} 7 / \mathrm{PD} 8$ & $1,25 \mathrm{~A} \mathrm{~b}$ & $154,71 \mathrm{~A} \mathrm{a}$ & $0,20 \mathrm{~A} \mathrm{a}$ & $0,86 \mathrm{~A} \mathrm{a}$ & $1,01 \mathrm{~A} \mathrm{a}$ & $8,68 \mathrm{~A} \mathrm{~b}$ & $1,22 \mathrm{~A} \mathrm{~b}$ & $7,47 \mathrm{~A} \mathrm{~b}$ \\
\hline PC5/PD4 & $1,06 \mathrm{~A} \mathrm{~b}$ & $127,21 \mathrm{~A} \mathrm{a}$ & $0,45 \mathrm{~A} \mathrm{a}$ & $1,14 \mathrm{~A} \mathrm{a}$ & $0,88 \mathrm{~A} \mathrm{a}$ & $9,50 \mathrm{~A} \mathrm{a}$ & $1,31 \mathrm{~A} \mathrm{~b}$ & $8,18 \mathrm{~A} \mathrm{a}$ \\
\hline PC3/PD3 & $1,21 \mathrm{~A} \mathrm{~b}$ & $182,53 \mathrm{~A} \mathrm{a}$ & $0,17 \mathrm{~A} \mathrm{a}$ & $0,96 \mathrm{~A} \mathrm{a}$ & $0,87 \mathrm{~A} \mathrm{a}$ & $9,90 \mathrm{~A} \mathrm{~b}$ & $0,87 \mathrm{~A} \mathrm{~b}$ & $9,03 \mathrm{~A} \mathrm{~b}$ \\
\hline CV ( \%) & 15,34 & 44,77 & 58,05 & 54,57 & 25,68 & 16,00 & 22,55 & 19,03 \\
\hline
\end{tabular}

CN: Cerrado nativo, PC3/PD3: plantio direto há três anos, PC5/PD4: plantio direto há quatro anos e PC7/PD8: plantio direto há oito anos. Médias seguidas da mesma letra não diferem entre si pelo teste de Tukey a $5 \%$. Letras maiúsculas comparam os tratamentos em cada profundidade e horizonte, e letras minúsculas comparam as profundidades e horizontes dentro do mesmo tratamento. 
entre os tratamentos foram observadas apenas na camada superficial, onde são mais intensos os processos de transformação da matéria orgânica pelos microrganismos do solo. Resultados semelhantes foram obtidos por D'Andréa et al. (2002) e Rosa et al. (2003) em estudo de Latossolos sob diferentes manejos, em comparação com o Cerrado. Segundo D'Andréa et al. (2002) e Santos et al. (2004), o valor de biomassa microbiana mais elevado no Cerrado nativo é reflexo de uma situação bastante particular para a microbiota do solo nesse sistema, que é estimulada pelo fornecimento contínuo de materiais orgânicos com diferentes graus de suscetibilidade à decomposição, originados da vegetação. Assim, as condições distintas do solo sob vegetação de Cerrado nativo, juntamente com a ausência de perturbações decorrentes de atividade antrópica, tornam possível a existência de maiores teores de biomassa microbiana, indicando o maior equilíbrio da microbiota do solo nesse ecossistema.

Os estoques de Cmic dos solos cultivados com PD tiveram reduções de 50 a $60 \%$, na camada de 0-5 cm, em relação ao CN. Essas reduções foram superiores às observadas pelos estoques de COT. Nesse sentido, confirma-se o Cmic como uma medida mais sensível às perdas de matéria orgânica relacionadas com o manejo, o que corrobora os resultados de Cardoso et al. (2009).

A relação $q$ Mic não apresentou efeito significativo entre os tratamentos, nem entre as profundidades. A contribuição do Cmic para o carbono orgânico total do solo variou, na camada de $0-5 \mathrm{~cm}$, de $1,62 \%$ no $\mathrm{CN}$ a $0,79-1,14 \%$ nos solos com PD. O maior valor, referente ao Cerrado, destacouse daqueles calculados para todos os demais tratamentos, ainda que as diferenças não tenham sido significativas. Essa relação reflete quanto do C orgânico está imobilizado na biomassa microbiana e mostra o potencial de reserva desse elemento no solo (Anderson \& Domsch, 1993). Nesse sentido, estatisticamente, as áreas apresentaram valores similares que indicam reserva de energia e nutrientes imobilizados na biomassa microbiana. Os maiores valores de $q \mathrm{Mic}$ indicam a maior conversão de COT em Cmic. Quando a biomassa do solo está sob algum fator de estresse, a capacidade de utilização do C é diminuída, reduzindo assim a relação $q$ Mic (Anderson \& Domsch, 1993). Segundo Gama-Rodrigues \& Gama-Rodrigues (2008), em solos com matéria orgânica de difícil decomposição a biomassa microbiana encontra-se sob estresse e é incapaz de utilizar totalmente o C orgânico; nesse caso, o $q$ Mic tende a diminuir.

Os teores de nitrogênio total (NT) na camada de $0-5 \mathrm{~cm}$ variaram de $1,99 \mathrm{~g} \mathrm{~kg}^{-1}$ no $\mathrm{CN}$ a 0,94-0,98 $\mathrm{g} \mathrm{kg}^{-1}$ nos solos cultivados sob PD.
$\mathrm{Na}$ camada de $5-10 \mathrm{~cm}$, os valores variaram de $1,12 \mathrm{~g} \mathrm{~kg}^{-1}$ no $\mathrm{CN}$ a $1,01-0,87 \mathrm{~g} \mathrm{~kg}^{-1}$ de solo nos outros tratamentos. Semelhantemente ao Cmic, as diferenças significativas entre os tratamentos restringiram-se à camada superficial $(0-5 \mathrm{~cm})$, não diferindo também entre as profundidades. Os maiores teores de NT foram encontrados no sistema mantido sob CN. A redução dos teores de NT nos tratamentos com os solos manejados foi de 51 a $53 \%$, quando comparados com o CN. Resultados semelhantes também foram encontrados por Santos et al. (2004) e Matias et al. (2009), que estudaram o efeito do manejo do solo nas propriedades biológicas em comparação com solo sem ação antrópica. Segundo Matias et al. (2009), a ausência de queimadas contribui para evitar as perdas de $\mathrm{N}$ por volatilização no Cerrado nativo - prática observada na conversão de área nativa em cultivada.

Quanto aos efeitos do PD na dinâmica da matéria orgânica, apenas o tratamento PC5/PD4 apresentou diferença significativa em relação ao CN quanto ao COT. Essa área foi cultivada cinco anos sob o sistema de plantio convencional. O revolvimento do solo ocasiona temporariamente melhores condições de aeração e maior exposição dos resíduos orgânicos incorporados; com isso, há rápida oxidação pelos microrganismos, reduzindo os teores de COT no solo (Albuquerque et al., 2005). Além disso, os quatro anos de implantação do sistema PD não proporcionaram aporte de matéria orgânica suficiente para elevar o COT ao nível do $\mathrm{CN}$. Observou-se no campo que a contribuição de matéria orgânica de um cultivo para o outro era incipiente, pois as condições climáticas favorecem a rápida decomposição desses resíduos, o que reforça a importância de um estudo sobre culturas mais resistentes à decomposição para formação de palhada nessa área.

Os tratamentos PC7/PD8 e PC3/PD3 não apresentaram diferença significativa quanto ao COT em relação ao $\mathrm{CN}$, devido ao maior tempo de aporte de matéria orgânica com implantação do PD no PC7/ PD8 (oito anos de PD) e ao menor tempo de uso do solo no PC3/PD3. É possível que os três anos de cultivos sob plantio convencional, a que foi submetida a área deste último tratamento, tenha sido suficiente para oxidar o CO dos compostos mais simples e facilmente decomponíveis, mas não das frações intermediárias e mais recalcitrantes, formadas sob Cerrado. Além disso, o não revolvimento do solo no PD preserva a sua estrutura, o que favorece uma maior proteção da matéria orgânica no interior dos agregados (Bayer et al., 2001).

Os valores da fração particulada (COP) diferiram estatisticamente entre o $\mathrm{CN}$ e os tratamentos com maior tempo de implantação do $\mathrm{PD}$, variando de 
$3,16 \mathrm{~g} \mathrm{~kg}^{-1}$ no $\mathrm{CN}$ a $2,18-2,20 \mathrm{~g} \mathrm{~kg}^{-1}$ nos tratamentos com $\mathrm{PC} 7 / \mathrm{PD} 8$ e $\mathrm{PC} 5 / \mathrm{PD} 4$, respectivamente, indicando que a condução do PD nessas áreas não elevou o COP ao que foi encontrado no CN. Além da deposição natural e constante de matéria orgânica no $\mathrm{CN}$, as condições mais ácidas do solo e os baixos teores de nutrientes conferem a este tratamento uma menor taxa de mineralização e transformação de frações mais grosseiras da matéria orgânica em frações mais ativas (Pragana, 2011). O COP é uma fração lábil e apresenta maior taxa de reciclagem dos constituintes orgânicos, de forma que as alterações em seus estoques pelo manejo do solo são percebidas geralmente em curto prazo (Bayer et al., 2001).

Os maiores valores de COP foram encontrados na camada de $0-5 \mathrm{~cm}$ de profundidade e apresentaram o mesmo padrão para todos os tratamentos, diminuindo em profundidade, similarmente ao comportamento do COT. Entretanto, comparando os três atributos, observa-se (Quadro 2) que o COP foi o que apresentou o menor teor. Segundo Roscoe \& Oliveira (2002), devido à reduzida superfície específica e densidade de carga superficial da areia, essa fração apresenta pouco ou nenhum material orgânico fortemente ligado, sendo pobre em complexos organominerais, tornando-se mais vulnerável à decomposição pelos microrganismos. O COP representou de 11 a $17 \%$ do COT, valores considerados baixos em comparação aos verificados em solos de outras regiões, em torno de $50 \%$ (Cambardella \& Elliot, 1992), mas que refletem as condições de temperatura e umidade altamente favoráveis à atividade microbiana nas condições de clima tropical quente e semiúmido do presente estudo. Conceição et al. (2005) e Nicoloso (2005), avaliando a qualidade do solo sob diferentes sistemas de manejo, constataram que a fração $\mathrm{COP}$ apresentou as maiores variações em função do manejo adotado, em relação ao COT.

Os valores da fração orgânica associada aos minerais $(\mathrm{COM})$ dos quatro tratamentos variaram de 83 a $89 \%$ do COT. Segundo Nicoloso (2005), mais de $80 \%$ do COT do solo é composto pela fração COM. Vários trabalhos têm demonstrado que a maior proporção da MOS está associada às frações mais finas (Freixo et al., 2002; Salton et al., 2005) e que a formação de complexos argilo-orgânicos auxilia na preservação da MOS.

Os valores do COM dos tratamentos com PD não apresentaram efeito significativo em relação ao $\mathrm{CN}$, porém diferiram entre si no horizonte $\mathrm{A}$, pois o tratamento PC3/PD3 apresentou o teor de COM estatisticamente mais elevado que o dos demais tratamentos com PD (Quadro 2). O solo deste tratamento está em uma área com menos tempo de uso, em relação às demais, e é possível que o tempo de cultivo a que foi submetido não tenha sido suficiente para oxidar o $\mathrm{CO}$ dos compostos das frações mais recalcitrantes, remanescentes do Cerrado nativo. Ademais, ainda há o aporte de matéria orgânica durante os três anos de PD. O COM normalmente é menos sensível às alterações de manejo, principalmente em curto prazo, sendo considerada a fração estável da matéria orgânica, composta, principalmente, por substâncias húmicas, que exercem papel significativo na estabilização dos microagregados (Cambardella \& Elliott, 1992). Segundo Salton et al. (2005), essa fração é considerada o estoque de $\mathrm{C}$ de médio e longo prazos.

\section{CONCLUSÕES}

1. O manejo imposto ao solo com cultivo convencional e posterior implantação do sistema plantio direto contribui para alterar as propriedades biológicas do solo em relação ao Cerrado nativo, notadamente no horizonte superficial.

2. A substituição do cerrado nativo por culturas anuais sob o sistema plantio direto reduz os teores de carbono da biomassa microbiana e de nitrogênio total e eleva a respiração basal nos tratamentos com três e quatro anos de implantação do plantio direto, indicando que esse tempo é insuficiente para melhoria desses atributos.

3. O sistema plantio direto mantém os estoque de $\mathrm{C}$ do solo ( $q \mathrm{Mic}$ ) em valores semelhantes aos do solo em condições naturais.

4. O aumento do tempo de uso do solo com plantio direto diminui o teor de $\mathrm{C}$ particulado e mantém o de $\mathrm{C}$ associado aos minerais, em comparação ao solo do Cerrado nativo.

5. O carbono da biomassa microbiana e o nitrogênio total são os atributos mais sensíveis às alterações no solo promovidas pela substituição do Cerrado nativo por culturas anuais sob plantio direto.

\section{LITERATURA CITADA}

ALBUQUERQUE, J.A.; MAFRA, A.L.; FONTOURA, S.M.V.; BAYER, C. \& PASSOS, J.F.M. Avaliação de sistemas de preparo e calagem em um Latossolo Bruno alumínico. R. Bras. Ci. Solo, 29:963-975, 2005.

ALEF, K. \& NANNIPIERI, P. Methods in applied microbiology and biochemistry. London, Academic Press, 1995. 576p.

ANDERSON, J.P.E. \& DOMSCH, K.H. The metabolic quotient (qCO2) as a specific activity parameter to assess the effects of environmental conditions, such as $\mathrm{pH}$, on the microbial biomass of forest soils. Soil Biol. Biochem., 25:393-395, 1993. 
BAYER, C.; MARTIN-NETO, L.; MIELNICZUK, J.; PILLON, C.N. \& SANGOI, L. Changes in soil organic matter fractions under subtropical no-till cropping systems. Soil Sci. Soc. Am. J., 65:1473-1478, 2001.

CALEGARI, A. Espécies para cobertura de solos. In: DAROLT, M.R., ed. Plantio direto: Pequena propriedade sustentável. Londrina, IAPAR, 1998. p.65-94. (Circular, 102)

CAMBARDELLA, C.A. \& ELLIOTT, E.T. Particulate soil organic matter change across a grassland cultivation sequence. Soil Sci. Soc. Am., 56:777-783, 1992.

CARDOSO, E.L.; SILVA, M.L.N.; MOREIRA, F.M.S. \& CURI, N. Atributos biológicos indicadores da qualidade do solo em pastagem cultivada e nativa no Pantanal. Pesq. Agropec. Bras., 44:631-637, 2009.

CHRISTENSEN, B.T. Organic matter in soil: Structure, function and turnover. Tjele, DIAS, 2000. 95p. (DIAS Report. Plant Production, 30)

COLLINS, H.P.; PAUL, E.A.; PAUSTIAN, K.; \& ELLIOT, E.T. Characterization of soil organic carbon relative to its stability and turnover. In: PAUL, E.A.; PAUSTIAN, K.; ELLIOT, E.T. \& COLE, C.V., eds. Soil organic matter in temperate agroecosystems, Long-term experiments in North America. Boca Raton, CRC Press, 1997. p15-49.

CONCEIÇÃO, P.C.; AMADO, T.J.C.; MIELNICZUK, J. \& SPAGNOLLO, E. Qualidade do solo em sistemas de manejo avaliada pela dinâmica da matéria orgânica e atributos relacionados. R. Bras. Ci. Solo, 29:777-788, 2005.

D’ANDRÉIA, A.F.; SILVA, M.L.N.; CURI, N.; SIQUEIRA, J.O. \& CARNEIRO, M.A.C. Atributos biológicos indicadores da qualidade do solo em sistemas de manejo na região do cerrado no sul do estado de Goiás. R. Bras. Ci. Solo, 26:913-923, 2002 .

FERREIRA, A.S.; CAMARGO, F.A.O. \& VIDOR, C. Utilização de microondas na avaliação da biomassa microbiana do solo. R. Bras. Ci. Solo, 23:991-996, 1999.

FERREIRA, E.A.B.; RESCK, D.V.S.; GOMES, A.C. \& RAMOS, M.L.G. Dinâmica do carbono da biomassa microbiana em cinco épocas do ano em diferentes sistemas de manejo do solo no cerrado. R. Bras. Ci. Solo, 31:16625-1635, 2007.

FIGUEREDO, C.C.; RESCK, D.V.S.; GOMES, A.C.; FERREIRA, E.A.B. \& RAMOS, M.L.G. Carbono e nitrogênio da biomassa microbiana em resposta a diferentes sistemas de manejo em um Latossolo Vermelho no cerrado. R. Bras. Ci. Solo, 31:551-562, 2007.

FREIXO, A.A.; MACHADO, P.L.O.A.; GUIMARÃES, C.M.; SILVA, C.A. \& FADIGAS, F.S. Estoques de carbono e nitrogênio e distribuição de frações orgânicas de Latossolo do Cerrado sob diferentes sistemas de cultivo. R. Bras. Ci. Solo, 26:425-434, 2002.

GAMA-RODRIGUES, E.F. \& GAMA-RODRGUES, A.C. Biomassa microbiana e ciclagem de nutrientes. In: SANTOS, G.A.; SILVA, L.S.; CANELLAS, L.P. \& CAMARGO, F.A.O., eds. Fundamentos da matéria orgânica do solo: Ecossistemas tropicais \& subtropicais. 2.ed. Porto Alegre, 2008. p.227-244.
HEIDE, D.M.; VITORINO, A.C.T.; TIRLONI, C. \& HOFFMANN, N.T.K. Frações orgânicas e estabilidade dos agregados de um Latossolo Vermelho Distroférrico sob diferentes usos. R. Ci. Agr., 51:143-160, 2009.

MATIAS, M.C.B.; SALVIANO, A.A.C.; LEITE, L.F.C. \& ARAÚJO, A.S.F. Biomassa microbiana e estoques de C e $\mathrm{N}$ do solo em diferentes sistemas de manejo, no Cerrado do Estado do Piauí. Acta Sci. Agron., 31:517-521, 2009.

NICOLOSO, R.S. Dinâmica da matéria orgânica do solo em áreas de integração lavoura-pecuária sob sistema plantio direto. Santa Maria, Universidade Federal de Santa Maria, 2005. 149p. (Tese de Mestrado)

PRAGANA, R.B. Caracterização pedológica e diagnóstico da qualidade de solos sob plantio direto na Serra do Quilombo, sudoeste piauiense. Recife, Universidade Federal Rural de Pernambuco, 2011. 159p. (Tese de Doutorado)

ROSA, M.E.C.; OLSZEVSKI, N.; MENDONÇA, E.S.; COSTA, L.M. \& CORREIA, J.R. Formas de carbono em Latossolo Vermelho Eutroférrico sob plantio direto no sistema biogeográfico do cerrado. R. Bras. Ci. Solo, 27:911-923, 2003.

ROSCOE, R. \& OLIVEIRA, P.L.A.M. Fracionamento físico do solo em estudos da matéria orgânica. Dourados, Embrapa Agropecuária Oeste; Rio de Janeiro, Embrapa Solos, 2002. $86 \mathrm{p}$.

SALTON, J.C.; MIELNICZUK, J.; BAYER, C.; FABRICIO, A.C.; MACEDO, M.C.M.; BROCH, D.L.; BOENI, M. \& CONEIÇÃO, P.C. Matéria orgânica do solo na integração lavoura-pecuária em Mato Grosso do Sul. Dourados, Embrapa Agropecuária do Oeste, 2005. 58p.

SANTOS, V.B.; CASTILHOS, D.D.; CASTILHOS, R.M.V.; PAULETTO, E.A.; GOMES, A.S. \& SILVA, D.G. Biomassa, atividade microbiana e teores de carbono e nitrogênio totais de um Planossolo sob diferentes sistemas de manejo. R. Bras. Agroci., 10:333-338, 2004.

SAS Institute INC. SAS/STAT user's guide. v. 9.0. Cary, 2002. $3 \mathrm{v}$.

SAVIOZZI, A.; BUFALINO, P.; LEVI-MINZI, R. \& RIFFALD, R. Biochemical activities in a degraded soil restored by two amendments: A laboratory study. Biol. Fert. Soils, 35:96-101, 2002.

TÓTOLA, M.R. \& CHAER, G.M. Microrganismos e processos microbiológicos como indicadores da qualidade dos solos. In: ALVAREZ V, V.H.; SCHAEFER, C.E.G.R.; BARROS, N.F.; MELLO, J.W.V. \& COSTA, L.M., eds. Tópicos em ciência do solo. Viçosa, MG, Sociedade Brasileira de Ciência do Solo, 2002. v.2. p.196-276.

TU, C.; RISTAINO, J.B. \& HU, S. Soil microbial biomass and activity in organic tomato farming systems: Effects of organic inputs and straw mulching. Soil Biol. Biochem., 38:247-255, 2006. 